\title{
Statistics of electric-quadrupole lines in atomic spectra
}

\author{
Jean-Christophe Pain 1 and Franck Gilleron \\ CEA, DAM, DIF, F-91297 Arpajon, France \\ Jacques Bauche and Claire Bauche-Arnoult \\ Laboratoire Aimé Cotton, Bâtiment 505, Campus d'Orsay, 91405 Orsay, \\ France
}

\begin{abstract}
In hot plasmas, a temperature of a few tens of $\mathrm{eV}$ is sufficient for producing highly stripped ions where multipole transitions become important. At low density, the transitions from tightly bound inner shells lead to electric-quadrupole lines which are comparable in strength with electric-dipole ones. In the present work, we propose analytical formulas for the estimation of the number of E2 lines in a transition array. Such expressions rely on statistical descriptions of electron states and $J$-levels. A generalized " $J$-file" sum rule for E2 lines and the strength-weighted shift and variance of the line energies of a transition array $n \ell^{N+1} \rightarrow n \ell^{N} n^{\prime} \ell^{\prime}$ of inter-configuration E2 lines are also presented.
\end{abstract}

\section{Introduction}

In atomic spectroscopy, the transition probabilities of electric-quadrupole (E2) lines are low, as compared to electric-dipole (E1) ones. Indeed, the ratio between E2 and E1 transition probabilities is proportional to $\left(a_{0} / \lambda Z^{*}\right)^{2}$, where $a_{0}$ is the Bohr radius, $Z^{*}$ the effective charge of the plasma and $\lambda$ the wavelength [1. This quantity is very small for most atomic transitions located in the range of UV to IR. However, they are important in astrophysics; for instance, Charro et al. performed a careful study of intensities of E2 spectral lines in the ion $\mathrm{Mg}$ II along the spectral series 2, 3. In astrophysical plasmas of Nebulae and of the interstellar medium, the density $n_{e}$ of the free electrons, which are responsible for collisional excitations and de-excitations, is generally very low, and the mean time between collisions is longer than radiative E2 lifetimes, even though these are much longer than for E1 transitions. Consequently, whenever an atom gets excited by a collision onto a level, metastable or not, its only decay channel is by radiative transitions (forbidden or allowed), and forbidden transitions appear with an intensity comparable to the allowed ones [4]. Fournier et al. [5] identified by ab initio calculations a bright $3 d^{8} \rightarrow 3 d^{7} 4 s$ E2 line for Mo XVII.

${ }^{1}$ jean-christophe.pain@cea.fr (corresponding author) 
They also studied the ratio of $3 d-4 s$ E2 to $3 d-4 p$ E1 emission in Mo XVI as a function of density, and noticed that the sharp falloff in the values of this ratio for Mo XVI above certain densities provides a plasma diagnostic for planned fusion reactors (high-Z impurities breed the dilution of the fusion "fuel" in reactors and can have a strong impact on spatial current distributions and radiative patterns in the plasma) 6, 7. Atomic data of tungsten are strongly needed for identification of emission lines in future fusion reactors (ITER) where it is used as a plasma-facing material 8 . Then, forbidden lines are of great interest for plasma diagnostics because the associated radiation intensity is very sensitive to density and temperature. Quinet gave a theoretical survey of forbidden transitions (E2 and M1) in the $4 p^{k}$ and $4 d^{k}$ ground configurations of ions Rh-like $W^{29+}$ to Ga-like $W^{43+}$ 9. Recently, Neu et al. pointed out a very strong E2 spectral line in the soft X-ray region at a wavelength of $0.793 \mathrm{~nm}$ originating from Ni-like tungsten $W^{46+}[10$. Clementson et al. measured this E2 line together with M3 (magnetic octupole) ground state transitions in the same ion using high-resolution crystal spectroscopy at the electron-beam ion trap (EBIT) facility in Livermore 11. EBIT light source was also used by Ralchenko et al. [12, 13, to record spectra of Br-like $W^{39+}$ to Co-like $W^{47+}$ in the $12-20 \mathrm{~nm}$ region. On the other hand, in most laboratory plasmas, $n_{e}$ is noticeably higher. For medium-range $n_{e}$, the probability for collisional depopulation may be surpassed only by the probability of radiative decay through allowed transitions. In this case, quadrupole transitions are not observed, justifying their name "forbidden". However, they can be important in some circumstances [14, 15, 16]. For instance, electric-quadrupole decays were observed in the spectra of neon-like [17] and nickel-like [18] ions produced by laser irradiation.

As mentioned before, forbidden lines can arise from metastable levels of excited configurations [19. In highly ionized atoms having ground configurations $3 p^{m}$ the lowest excited configuration is $3 p^{m-1} 3 d$. For $m>1, p^{m-1} d$ has levels with $J^{\prime}$ greater by two than the largest $J$ of $p^{m}$, and downward electric-dipole transitions from those levels are therefore forbidden by the dipole selection rule $|\Delta J| \leq 1$. Magnetic-dipole and electric-quadrupole transitions within the configuration Fe IX $3 p^{5} 3 d$ and arising from such metastable levels are responsible for several lines of the solar corona. It is interesting to mention that in $\mathrm{Pb} \mathrm{I}$, parity-forbidden transitions for electric-dipole radiation $6 p^{2} \rightarrow 6 p 7 p$ have been observed [19. In that case, the upper levels are not metastable, because E1 transitions to $6 p 7 s$ are possible. In absorption, electric-quadrupole transitions of the type $s \rightarrow d$ have been extensively studied in the alkalis [20, 21].

The effective core charge (or screened nuclear charge) seen by an electron is the net charge $Z$ of the nucleus with the $N_{c}-1$ electrons of the ion core $Z_{c}=Z-N_{c}+1$. Along an iso-electronic sequence, the $\mathrm{E} 2$ radial matrix elements decrease like $Z_{c}^{-2}$, rather than like $Z_{c}^{-1}$ as do the E1 matrix elements. For inter-configuration transitions with $\Delta n \neq 0$, one has $A_{\mathrm{E}_{2}} / A_{\mathrm{E}_{1}} \propto Z_{c}^{2}$, where $A_{\mathrm{E}_{1}}$ and $A_{\mathrm{E}_{2}}$ represent the transition probability rates for E1 and E2 lines respectively [19, 22. For inter-configuration transitions with $\Delta n=0$, the orders of magnitudes of the E1 and E2 transition probabilities vary in the same way with respect to the effective core charge $Z_{c}$ [19.

The detailed calculation of all the line energies and strengths in complex atomic spectra is difficult and, in some circumstances, useless. Indeed, when the densiy is sufficiently high so that the physical broadening mechanisms (e.g. Stark shifts) are important and/or when the number of lines becomes large, 
the lines coalesce into broad structures. Statistical methods 223, 24, 25, 26. 27, 28, 29, 30, 31, 32, 33 are required because, experimentally, some quantities cannot be determined individually, but only as weighted average quantities (for instance, in emission/absorption spectra of highly ionized atoms). Moreover, explicit quantum calculations can be unappropriate, e.g. if the hamiltonian matrix is huge. In addition, global methods can reveal physical properties hidden in a detailed treatment of levels and lines. For example, they have opened the way to the definition of the generalized $J$-file sum rule 34 and of effective temperatures for the configuration populations [35].

Even for E1 lines, there exists no general compact formula for the exact number of lines in a transition array. Using group-theoretical methods, Krasnitz 36, 37 obtained such a compact formula only in the simple case of configurations built with non-equivalent electrons. The statistics of E1 lines was studied by Bauche and Bauche-Arnoult [28, 38, but very few results were obtained as concerns the statistics of E2 lines in complex atomic spectra. The purpose of the present work is to provide an analytical formula for the estimation of the number of E2 lines in a transition array. Such a quantity is important for opacity codes, for instance, in order to decide whether a transition array can be described statistically 24, 25, 26, 27 or requires a DLA (Detailed-Line Accounting) calculation, relying on the diagonalization of the Hamiltonian [39]. Like for the E1 case, a statistical description is used, because this problem does not lend itself readily to exact calculations. The first step consists in determining the statistics of the angular quantum number $J$. However, due to the fact that the quantum number $J$ is the eigenvalue of no simple operator, its mathematical study is tedious. Therefore, it is more appropriate to study the distribution of $M$, the eigenvalue (in units of $\hbar$ ) of the operator $J_{z}$ (projection along the $z$-axis of operator $\vec{J}$ ). The $J$ values can be obtained from the $M$ values by means of the method of Condon and Shortley 40. Any unknown distribution can be characterized by its moments. The $n^{t h}$ moment of the $M$ distribution reads:

$$
\mu_{n}=\frac{1}{g} \sum_{\gamma J M}\left\langle\gamma J M\left|J_{z}\right| \gamma J M\right\rangle^{n},
$$

where the sum runs over all the $g$ states of the configuration in intermediate coupling. The distribution $P(M)$ of $M$ being symmetrical, the odd-order moments are zero. In general, it is admitted 41 that the first four moments are sufficient to capture the shape of a distribution.

In section 2, the second and fourth-order moments of $P(M)$ for a nonrelativistic configuration are recalled. Section 3 contains the calculation of the number of E2 lines between two configurations in the case of three different modelings of $P(M)$ : Gaussian, Gram-Charlier and Generalized Gaussian. Section 4 contains the calculation, using the aforementioned three distributions, of the number of E2 lines inside a configuration. In section [5, a generalized $J$-file sum rule for E2 lines is given, and in section 6 the expressions of the strengthweighted shift and variance of a transition array of inter-configuration E2 lines are provided for $n \ell^{N+1} \rightarrow n \ell^{N} n^{\prime} \ell^{\prime}$. Section 7 is the conclusion. 


\section{Variance and kurtosis of the projection of the $J$ angular momentum}

The variance $v$ of the states of configuration $\ell^{N}$ is given by [32, 38]:

$$
v\left(\ell^{N}\right)=\mu_{2}\left(\ell^{N}\right)=\frac{N(4 \ell+2-N)}{4 \ell+1} v(\ell),
$$

where

$$
v(\ell)=\frac{4 \ell^{2}+4 \ell+3}{12} .
$$

The fourth-order moment of configuration $\ell^{N}$ reads

$$
\mu_{4}\left(\ell^{N}\right)=N(4 \ell+2-N)[x(\ell) N(4 \ell+2-N)+y(\ell)]
$$

where

$$
x(\ell)=\frac{2 \ell-1}{240\left(16 \ell^{2}-1\right)}\left(40 \ell^{3}+84 \ell^{2}+110 \ell+51\right)
$$

and

$$
y(\ell)=\frac{2 \ell+1}{60\left(16 \ell^{2}-1\right)}\left(-16 \ell^{4}-24 \ell^{3}-8 \ell^{2}+24 \ell+9\right) .
$$

For a configuration with $w$ open sub-shells $\ell_{1}^{N_{1}} \ell_{2}^{N_{2}} \ell_{3}^{N_{3}} \cdots \ell_{w}^{N_{w}}$, one has for the total variance:

$$
v\left(\ell_{1}^{N_{1}} \ell_{2}^{N_{2}} \ell_{3}^{N_{3}} \cdots \ell_{w}^{N_{w}}\right)=\sum_{i=1}^{w} v\left(\ell_{i}^{N_{i}}\right)
$$

and for the total kurtosis:

$$
\begin{aligned}
\mu_{4}\left(\ell_{1}^{N_{1}} \ell_{2}^{N_{2}} \ell_{3}^{N_{3}} \cdots \ell_{w}^{N_{w}}\right)= & \sum_{i=1}^{w} \mu_{4}\left(\ell_{i}^{N_{i}}\right) \\
& +6 \sum_{i, j=1, j>i}^{w} \mu_{2}\left(\ell_{i}^{N_{i}}\right) \mu_{2}\left(\ell_{j}^{N_{j}}\right)
\end{aligned}
$$

where the first sum runs over all subshells $i$ of interest, and the second sum runs over all pairs $(i, j)$ of subshells. In the following section, we will use the fourth-order reduced moment:

$$
\alpha_{4}=\frac{\mu_{4}}{v^{2}},
$$

which can also be put in the form [42]:

$$
\alpha_{4}=3-\frac{1}{v^{2}} \sum_{i=1}^{w} q_{i}\left(a_{i}+q_{i} b_{i}\right)
$$

where 


$$
\begin{gathered}
a_{i}=\frac{\left(2 \ell_{i}+1\right)\left(16 \ell_{i}^{4}+24 \ell_{i}^{3}+8 \ell_{i}^{2}-24 \ell_{i}-9\right)}{60\left(4 \ell_{i}-1\right)}, \\
b_{i}=\frac{-16 \ell_{i}^{4}-16 \ell_{i}^{3}+88 \ell_{i}^{2}+136 \ell_{i}+3}{120\left(4 \ell_{i}-1\right)}
\end{gathered}
$$

and

$$
q_{i}=\frac{N_{i}\left(4 \ell_{i}+2-N_{i}\right)}{4 \ell_{i}+1} .
$$

\section{$3 \quad$ Number of E2 lines between two different configurations}

The initial and final configurations are denoted $C$ and $C^{\prime}$ respectively. The selection rules for E2 transitions in intermediate coupling are $\Delta J=0, \pm 1, \pm 2$ with $J+J^{\prime} \geq 2$, and $\Delta \ell=0, \pm 2$ with $\ell+\ell^{\prime} \geq 2$ (transitions $s \rightarrow s^{\prime}$ are strictly forbidden for isolated atoms, but have been observed by means of Stark-effectinduced mixing of $n^{\prime} s^{\prime}$ with $n^{\prime \prime} p$ states [43]). Therefore, the number $L_{E_{2}}$ of E2 lines between two different configurations is given by

$$
\begin{aligned}
L_{E_{2}}\left(C-C^{\prime}\right)= & \sum_{J=J_{\min }}^{\infty} Q_{C}(J)\left[Q_{C^{\prime}}(J)+Q_{C^{\prime}}(J+1)\right. \\
& \left.+Q_{C^{\prime}}(J-1)+Q_{C^{\prime}}(J+2)+Q_{C^{\prime}}(J-2)\right] \\
& +\epsilon^{\prime}\left(J_{\min }\right),
\end{aligned}
$$

where $Q_{C}(J)$ (respectively $Q_{C^{\prime}}(J)$ ) is the number of the levels of configuration $C$ (respectively $C^{\prime}$ ) with total angular momentum $J$ and $\epsilon\left(J_{\min }\right)$ is a small correction, $J_{\min }$ being the smallest value of $J$. In many cases, $J_{\min }=0$ or $1 / 2$ according to the parity of the number of electrons. For configuration $s d, J_{\min }=1$ and for configurations involving orbitals with a large angular momentum $J$, the value of $J_{\min }$ can be larger. A continuous representation of Eq. (14) yields

$$
\begin{aligned}
L_{E_{2}}\left(C-C^{\prime}\right) \approx & \int_{-1 / 2}^{\infty} Q_{C}(J)\left[Q_{C^{\prime}}(J)+Q_{C^{\prime}}(J+1)\right. \\
& \left.+Q_{C^{\prime}}(J-1)+Q_{C^{\prime}}(J+2)+Q_{C^{\prime}}(J-2)\right] d J \\
& +\epsilon^{\prime}\left(J_{\min }\right),
\end{aligned}
$$

where $\epsilon^{\prime}\left(J_{\min }\right)$ is a border correction. In fact, $\epsilon^{\prime}(0)=\epsilon^{\prime}\left(\frac{1}{2}\right)=0$ are values compatible with the accuracy of the replacement of a discrete sum by an integral (see Appendix A). Using the second-order Taylor development

$$
Q(J+k) \approx Q(J)+\left.k \frac{d Q}{d J}\right|_{J}+\left.\frac{k^{2}}{2} \frac{d^{2} Q}{d J^{2}}\right|_{J}
$$

one obtains 


$$
L_{E_{2}}\left(C-C^{\prime}\right) \approx 5 \int_{-1 / 2}^{\infty} Q_{C}(J)\left[Q_{C^{\prime}}(J)+\frac{d^{2} Q_{C^{\prime}}}{d J^{2}}\right] d J
$$

The method of Condon and Shortley [40] enables one to express $Q(J)$ as

$$
Q(J)=\sum_{M=J}^{M=J+1}(-1)^{J-M} P(M)=P(J)-P(J+1),
$$

where $P$ represents the distribution of the angular-momentum projection $M$. For a configuration $\ell_{1}^{N_{1}} \ell_{2}^{N_{2}} \ell_{3}^{N_{3}} \cdots \ell_{w}^{N_{w}}, P(M)$ is determined through the relation

$$
P_{N_{1}, N_{2}, \cdots}(M)=\left(P_{N_{1}} \otimes P_{N_{2}} \otimes P_{N_{3}} \otimes \cdots \otimes P_{N_{w}}\right)(M),
$$

where the distributions are convolved two at a time, which means that

$$
\left(P_{N_{i}} \otimes P_{N_{j}}\right)(M)=\sum_{M^{\prime}=-\infty}^{+\infty} P_{N_{i}}\left(M^{\prime}\right) \times P_{N_{j}}\left(M-M^{\prime}\right) .
$$

The total number $L_{E_{2}}\left(C-C^{\prime}\right)$ of E2 lines is invariant under the following transformations:

(i) The configurations $C$ and $C^{\prime}$ can be interchanged.

(ii) In $C$ and $C^{\prime}$, one can replace simultaneously all the subshells by their complementary subshells, e.g.: $\ell^{N}$ by $\ell^{4 \ell+2-N}$. Therefore, the $C \rightarrow C^{\prime}$ array, denoted in general $\ell_{1}^{N_{1}+1} \ell_{2}^{N_{2}} \rightarrow \ell_{1}^{N_{1}} \ell_{2}^{N_{2}+1}$ has the same number of E2 lines as its complementary array:

$$
\ell_{1}^{4 \ell_{1}+1-N_{1}} \ell_{2}^{4 \ell_{2}+2-N_{2}} \rightarrow \ell_{1}^{4 \ell_{1}+2-N_{1}} \ell_{2}^{4 \ell_{2}+1-N_{2}}
$$

(iii) The $C \rightarrow C^{\prime}$ transition array has also the same number of E2 lines as its two following semi-complementary array

$$
\ell_{1}^{4 \ell_{1}+1-N_{1}} \ell_{2}^{N_{2}+1} \rightarrow \ell_{1}^{4 \ell_{1}+2-N_{1}} \ell_{2}^{N_{2}}
$$

and

$$
\ell_{1}^{N_{1}} \ell_{2}^{4 \ell_{2}+2-N_{2}} \rightarrow \ell_{1}^{N_{1}+1} \ell_{2}^{4 \ell_{2}+1-N_{2}} .
$$

The first semi-complementary array is deduced from $C \rightarrow C^{\prime}$ as follows: replace the $\ell_{1}$ subshells by their complementaries, and exchange the $\ell_{2}$ subshells. For the second one, replace the $\ell_{2}$ subshells by their complementaries, and exchange the $\ell_{1}$ subshells. Complementarity ensures that the variances are unchanged, but it is not the case for semi-complementarity. However, since

$$
\begin{aligned}
& v\left(\ell_{1}^{4 \ell_{1}+1-N_{1}}\right)+v\left(\ell_{2}^{N_{2}+1}\right)+v\left(\ell_{1}^{4 \ell_{1}+2-N_{1}}\right)+v\left(\ell_{2}^{N_{2}}\right) \\
= & v\left(\ell_{1}^{N_{1}+1}\right)+v\left(\ell_{2}^{N_{2}}\right)+v\left(\ell_{1}^{N_{1}}\right)+v\left(\ell_{2}^{N_{2}+1}\right),
\end{aligned}
$$


semi-complementarity can be ensured by replacing $v_{C}$ and $v_{C^{\prime}}$ by their half sum $\left(v_{C}+v_{C^{\prime}}\right) / 2$ [38. However, the kurtosis does not follow a relation similar to Eq. (24). It can only be ensured that the final formula for $L_{E_{2}}\left(C-C^{\prime}\right)$ be symmetrical in $C$ and $C^{\prime}$, by setting $\alpha_{4}=\left(\alpha_{4, C}+\alpha_{4, C^{\prime}}\right) / 2$. Following [38, we make the assumption that

$$
Q(J) \approx-\left.\frac{d P}{d M}\right|_{J+1 / 2} .
$$

\begin{tabular}{|c|c|c|c|c|}
\hline \hline Transition array & Exact & Gaussian & Gram-Charlier & GG \\
\hline \hline$d^{4} \rightarrow d^{3} s$ & 887 & $\begin{array}{c}953 \\
(+7.44 \%)\end{array}$ & $\begin{array}{c}885 \\
(-0.23 \%)\end{array}$ & $\begin{array}{c}839 \\
(-5.41 \%)\end{array}$ \\
\hline$d^{3} \rightarrow d^{2} g$ & 1015 & $\begin{array}{c}1215 \\
(+19.70 \%)\end{array}$ & $\begin{array}{c}1092 \\
(+7.56 \%)\end{array}$ & $\begin{array}{c}1045 \\
(+2.96 \%)\end{array}$ \\
\hline$p^{3} \rightarrow p^{2} f$ & 110 & $\begin{array}{c}124 \\
(+12.73 \%)\end{array}$ & $\begin{array}{c}116 \\
(+5.45 \%)\end{array}$ & $\begin{array}{c}80 \\
(-27.27 \%)\end{array}$ \\
\hline$p^{4} \rightarrow p^{3} f$ & 110 & $\begin{array}{c}124 \\
(+12.73 \%)\end{array}$ & $\begin{array}{c}116 \\
(+5.45 \%)\end{array}$ & $\begin{array}{c}81 \\
(-26.36 \%)\end{array}$ \\
\hline$d^{5} \rightarrow d^{4} g$ & 8299 & $\begin{array}{c}9550 \\
(+15.07 \%)\end{array}$ & $\begin{array}{c}8697 \\
(+4.80 \%)\end{array}$ & $\begin{array}{c}8415 \\
(+0.14 \%)\end{array}$ \\
\hline$d^{5} g^{3} \rightarrow d^{4} g^{4}$ & 296780266 & 299065925 \\
& & 298981701 & 293198984 \\
$(+0.77 \%)$ & $(+0.74 \%)$ & $(-1.21 \%)$ \\
\hline$d^{4} i^{1} \rightarrow d^{3} g^{1} i^{1}$ & 762544 & 897641 & 783942 & 774216 \\
& & $(+17.72 \%)$ & $(+2.81 \%)$ & $(+1.54 \%)$ \\
\hline \hline
\end{tabular}

Table 1: Number of E2 lines for different inter-configuration transitions calculated using Gaussian, fourth-order Gram-Charlier and Generalized-Gaussian modelings of $P(M)$ and compared to the exact values. An orbital $i$ corresponds to $\ell=6$.

Considering the Gaussian modeling of $P(M)$ :

$$
P(M)=\frac{g}{\sqrt{2 \pi v}} \exp \left(-\frac{M^{2}}{2 v}\right),
$$

one obtains

$$
Q(J)=\frac{g}{v \sqrt{8 \pi v}}(2 J+1) \exp \left(-\frac{(2 J+1)^{2}}{8 v}\right),
$$

which yields

$$
L_{E_{2}}\left(C-C^{\prime}\right)=\frac{5 g_{C} g_{C^{\prime}}(2 v-3)}{16 \sqrt{\pi} v^{5 / 2}},
$$

where $g_{C}$ represents the degeneracy (total number of states) of configuration $C$; for instance, the degeneracy of a configuration $\ell_{1}^{N_{1}} \ell_{2}^{N_{2}} \ell_{3}^{N_{3}} \cdots \ell_{w}^{N_{w}}$ reads

$$
g_{C}=\prod_{i=1}^{w}\left(\begin{array}{c}
4 \ell_{i}+2 \\
N_{i}
\end{array}\right) .
$$


It was also suggested in Ref. 38 to use a fourth-order Gram-Charlier modeling of $P(M)$ :

$$
P(M)=\frac{g}{\sqrt{2 \pi v}} \exp \left(-\frac{M^{2}}{2 v}\right)\left[1+\frac{\left(\alpha_{4}-3\right)}{24}\left(3-6 \frac{M^{2}}{v}+\frac{M^{4}}{v^{2}}\right)\right] .
$$

In that case, one finds

$$
\begin{aligned}
Q(J)= & \frac{g}{v \sqrt{8 \pi v}}\left[(2 J+1)+\frac{\left(\alpha_{4}-3\right)}{24}(15(2 J+1)\right. \\
& \left.\left.-10 \frac{(2 J+1)^{3}}{4 v}+\frac{(2 J+1)^{5}}{16 v^{2}}\right)\right] \exp \left(-\frac{(2 J+1)^{2}}{8 v}\right),
\end{aligned}
$$

which yields

$$
\begin{aligned}
L_{E_{2}}\left(C-C^{\prime}\right)= & \frac{1}{16384 \sqrt{\pi} v^{5 / 2}}\left[5 g_{C} g_{C^{\prime}}(-6747+2018 v\right. \\
& \left.\left.+5 \alpha_{4}\left(938-124 v+21 \alpha_{4}(-11+2 v)\right)\right)\right] .
\end{aligned}
$$

Finally, considering the Generalized-Gaussian modeling of $P(M)$ :

$$
P(M)=\frac{g}{\sqrt{v}} \frac{e^{-\left|\frac{M}{\lambda \sqrt{v}}\right|^{\nu}}}{2 \lambda \Gamma\left(1+\frac{1}{\nu}\right)} \quad \text { with } \quad \lambda=\sqrt{\frac{\Gamma\left(\frac{1}{\nu}\right)}{\Gamma\left(\frac{3}{\nu}\right)}},
$$

where $\nu$ is a positive real number, and $\Gamma(x)$ is the ordinary gamma function, one has

$$
Q(J)=\frac{g_{C} \nu}{2 \lambda^{2} v \Gamma\left(1+\frac{1}{\nu}\right)}\left(\frac{2 J+1}{2 \lambda \sqrt{v}}\right)^{\nu-1} \exp \left(-\left[\frac{2 J+1}{2 \lambda \sqrt{v}}\right]^{\nu-1}\right)
$$

and the resulting number of $\mathrm{E} 2$ lines is

$$
\begin{aligned}
L_{E_{2}}\left(C-C^{\prime}\right)= & \frac{5 g_{C} g_{C^{\prime}} \nu^{3} 2^{\frac{1}{\nu}}}{64 \lambda^{3} v^{3 / 2} \Gamma\left(\frac{1}{\nu}\right)^{2}}\left[-\frac{4^{\frac{1}{\nu}}(\nu-1)(2 \nu-1)}{\lambda^{2} v} \Gamma\left(2-\frac{3}{\nu}\right)\right. \\
& \left.+4 \Gamma\left(2-\frac{1}{\nu}\right)\right] .
\end{aligned}
$$

As can be seen in table 1, the fourth-order Gram-Charlier expansion series provides a better agreement in most of the cases, except for $d^{4} i^{1} \rightarrow d^{3} g^{1} i^{1}$ and $d^{5} \rightarrow d^{4} g^{1}$. This is due to the fact that, when a high- $\ell$ electron is involved, the shape of the distribution $P(M)$ is not quasi-Gaussian anymore, but exhibits a plateau 42, which cannot be modeled by Gram-Charlier expansion. On the other hand, the Generalized Gaussian can depict such situations (the "door function" corresponds to an exponent equal to $\nu=9 / 8$ ). Furthermore, the second-order Taylor-series expansion (see Eq. (16)) is not as precise as in the E1 case, since the selection rules here imply that the maximum value of $k$ is 2 (against 1 in the $\mathrm{E} 1$ case). 


\section{Number of E2 lines in the intra-configuration case $\left(C=C^{\prime}\right)$}

\begin{tabular}{|c|c|c|c|c|}
\hline \hline Configuration & Exact & Gaussian & Gram-Charlier & GG \\
\hline \hline$d^{6}$ & 358 & $\begin{array}{c}380 \\
(+6.15 \%)\end{array}$ & $\begin{array}{c}352 \\
(-1.68 \%)\end{array}$ & $\begin{array}{c}335 \\
(-6.42 \%)\end{array}$ \\
\hline$d^{5} p$ & 15383 & $\begin{array}{c}16499 \\
(+7.25 \%)\end{array}$ & $\begin{array}{c}15446 \\
(+0.41 \%)\end{array}$ & $\begin{array}{c}14967 \\
(-2.70 \%)\end{array}$ \\
\hline$p^{2} f$ & 353 & $\begin{array}{c}402 \\
(+13.88 \%)\end{array}$ & $\begin{array}{c}358 \\
(+1.42 \%)\end{array}$ & $\begin{array}{c}322 \\
(-8.78 \%)\end{array}$ \\
\hline$p^{3} f$ & 611 & $\begin{array}{c}696 \\
(+13.91 \%)\end{array}$ & $\begin{array}{c}622 \\
(+1.80 \%)\end{array}$ & $\begin{array}{c}567 \\
(-7.20 \%)\end{array}$ \\
\hline$g^{7}$ & 903622 & 968412 & 909392 & 892418 \\
& & $(+7.17 \%)$ & $(+0.64 \%)$ & $(-1.24 \%)$ \\
\hline$f^{7} p^{2}$ & 5437574 & 5839649 & 5481760 & 5373233 \\
& & $(+7.39 \%)$ & $(+0.81 \%)$ & $(-1.18 \%)$ \\
\hline$f^{7} d^{2}$ & 40832855 & 43424047 \\
& & $\begin{array}{c}41104770 \\
(+6.35 \%)\end{array}$ & $\begin{array}{c}40379223 \\
(-1.11 \%)\end{array}$ \\
\hline$f^{3} p^{2}$ & 97615 & 107207 & 98683 & 96137 \\
& & $(+9.83 \%)$ & $(+1.09 \%)$ & $(-1.51 \%)$ \\
\hline$f^{3} d^{2}$ & 690870 & $\begin{array}{c}746580 \\
(+0.81 \%)\end{array}$ & $\begin{array}{c}696669 \\
(+0.84 \%)\end{array}$ & $\begin{array}{c}681553 \\
(-1.35 \%)\end{array}$ \\
\hline$d^{2} l$ & 2376 & 2545 & 2064 & 2365 \\
& & $(+7.11 \%)$ & $(-13.13 \%)$ & $(-0.46 \%)$ \\
\hline$p^{3} i$ & 616 & 655 & 529 & 581 \\
& & $(+6.33 \%)$ & $(-14.12 \%)$ & $(-5.68 \%)$ \\
\hline \hline
\end{tabular}

Table 2: Number of E2 lines inside different configurations calculated using Gaussian, fourth-order Gram-Charlier and Generalized-Gaussian modelings of $P(M)$ and compared to the exact values. An orbital $l$ corresponds to $\ell=8$.

The number of E2 lines inside a configuration can be estimated as

$$
L_{E_{2}} \approx \int_{-1 / 2}^{\infty} Q(J)\left[\frac{1}{2}(Q(J)-1)+Q(J+1)+Q(J+2)\right] d J .
$$

Using the second-order Taylor development presented in Eq. (16), one finds the approximate expression

$$
L_{E_{2}} \approx \frac{1}{2} \int_{-1 / 2}^{\infty} Q(J)\left[5\left\{Q(J)+\frac{d^{2} Q}{d J^{2}}\right\}+6 \frac{d Q}{d J}-1\right] d J
$$

A Gaussian expression of $P(M)$ (see Eq. (26) ) leads to

$$
L_{E_{2}}=\frac{g_{C}\left(-8 \sqrt{2} v_{C}^{2}+5 g_{C}\left(2 v_{C}-3\right)\right)}{32 \sqrt{\pi} v_{C}^{5 / 2}},
$$

and a fourth-order Gram-Charlier expression of $P(M)$ (see Eq. (30) ) to 


$$
\begin{aligned}
L_{E_{2}}= & \frac{-2048 g_{C}\left(\alpha_{4, C}+5\right) v_{C}^{2}}{32768 \sqrt{2 \pi} v_{C}^{5 / 2}} \\
& +\frac{5 \sqrt{2} g_{C}^{2}}{32768 \sqrt{2 \pi} v_{C}^{5 / 2}}\left\{-6747+2018 v_{C}+5 \alpha_{4, C}\left[938-124 v_{C}\right.\right. \\
& \left.\left.+21 \alpha_{4, C}\left(2 v_{C}-11\right)\right]\right\} .
\end{aligned}
$$

Using a Generalized-Gaussian expression of $P(M)$ (see Eq. (33)), one obtains

$$
\begin{aligned}
L_{E_{2}}= & \frac{5 g_{C}^{2} \nu^{3} 2^{1 / \nu}}{128 \lambda^{3} v_{C}^{3 / 2} \Gamma\left(\frac{1}{\nu}\right)^{2}}\left[-\frac{4^{1 / \nu}(\nu-1)(2 \nu-1)}{\lambda^{2} v_{C}} \Gamma\left(2-\frac{3}{\nu}\right)\right. \\
& \left.+4 \Gamma\left(2-\frac{1}{\nu}\right)\right]-\frac{\nu g_{C}}{4 \lambda \sqrt{v_{C}} \Gamma\left(\frac{1}{\nu}\right)} .
\end{aligned}
$$

Here also the fourth-order Gram-Charlier expansion series provides a better agreement in most of the cases, except for configurations with high- $\ell$ electrons (see table 2). The results are closer to the exact values in the intra-configuration case than in the inter-configuration case. This is due to the fact that the number of lines in the intra-configuration case is evaluated with the exact variance and the exact kurtosis of the distribution of $M$ (in the inter-configuration case, they were obtained as the arithmetic average over the initial and final configurations).

It is worth mentioning that global methods were also used for the study of statistical properties of Auger amplitudes and rates [4] and the dispersion of gyromagnetic ratios in complex spectra [45]. In appendix C, we give, following the work of Kynienè et al. [44], an approximate expression for the number of Auger amplitudes with the three different modelings of $P(M)$.

\section{A generalized $J$-file sum rule for E2 lines}

Using the second-quantization technique [46], Bauche et al. 34] established a linear relationship between the angular coefficient of the exchange Slater integral $G^{1}\left(n \ell, n^{\prime} \ell-1\right)$ and the $J$-file sums of the E1 line strengths defined by Condon and Shortley [40. In the case of E2 lines, a $J$-file sum rule can be also obtained, following the same procedure as for E1 lines. The sum of the strengths of all E2 lines starting from a level $\gamma J$ of the upper configuration in the $\ell^{N} \ell^{\prime N^{\prime}+1} \rightarrow$ $\ell^{N+1} \ell^{\prime N^{\prime}}$ array is given by:

$$
\begin{aligned}
S_{E 2}\left[\left(\ell^{N} \ell^{\prime N^{\prime}+1}\right) \gamma J-\ell^{N+1} \ell^{\prime N^{\prime}}\right]= & (2 J+1)\left[\frac{\left(N^{\prime}+1\right)}{\left(2 \ell^{\prime}+1\right)}\left\langle\ell\left\|C^{(2)}\right\| \ell^{\prime}\right\rangle^{2}\right. \\
& \left.+C\left(G^{2} ; \gamma J\right)\right]\left[I\left(n \ell, n^{\prime} \ell^{\prime}\right)\right]^{2}
\end{aligned}
$$

with

$$
I\left(n \ell, n^{\prime} \ell^{\prime}\right)=\int_{0}^{\infty} R_{n \ell}(r) r^{2} R_{n^{\prime} \ell^{\prime}}(r) d r
$$


and

$$
\left\langle\ell|| C^{(2)}|| \ell^{\prime}\right\rangle=(-1)^{\ell} \sqrt{(2 \ell+1)\left(2 \ell^{\prime}+1\right)}\left(\begin{array}{ccc}
\ell & 2 & \ell^{\prime} \\
0 & 0 & 0
\end{array}\right) .
$$

The quantity $C\left(G^{2} ; \gamma J\right)$ represents the coefficient of the $G^{2}\left(n \ell, n^{\prime} \ell^{\prime}\right)$ Slater integral in the electrostatic energy of the level $\gamma J$. In the present case, one has, if $\left|\ell^{\prime}-\ell\right|=2$ and $\ell_{>}=\max \left(\ell, \ell^{\prime}\right)$ :

$$
\left\langle\ell|| C^{(2)} \| \ell^{\prime}\right\rangle^{2}=\frac{3 \ell_{>}\left(\ell_{>}-1\right)}{2\left(2 \ell_{>}-1\right)},
$$

and if $\ell=\ell^{\prime}$ :

$$
\left\langle\ell\left\|C^{(2)}\right\| \ell^{\prime}\right\rangle^{2}=\frac{\ell(\ell+1)(2 \ell+1)}{(2 \ell-1)(2 \ell+3)} .
$$

These results remain unchanged if passive subshells are added to both configurations. It is interesting to mention that the total strength of transition array $C \rightarrow C^{\prime}$ with $C=n \ell^{N+1} n^{\prime} \ell^{\prime N^{\prime}} n^{\prime \prime} \ell^{\prime \prime N^{\prime \prime}} \ldots$ and $C^{\prime}=n \ell^{N} n^{\prime} \ell^{\prime N^{\prime}+1} n^{\prime \prime} \ell^{N^{\prime \prime}} \ldots$ reads

$$
T\left(C \rightarrow C^{\prime}\right)=2 \frac{(N+1)\left(4 \ell^{\prime}+2-N^{\prime}\right)}{(4 \ell+2)\left(4 \ell^{\prime}+2\right)} g_{C}\left\langle\ell\left\|C^{(2)}\right\| \ell^{\prime}\right\rangle^{2}\left[I\left(n \ell, n^{\prime} \ell^{\prime}\right)\right]^{2},
$$

where $g_{C}$ represents the degeneracy of configuration C, i.e.

$$
g_{C}=\left(\begin{array}{c}
4 \ell+2 \\
N+1
\end{array}\right)\left(\begin{array}{c}
4 \ell^{\prime}+2 \\
N^{\prime}
\end{array}\right)\left(\begin{array}{c}
4 \ell^{\prime \prime}+2 \\
N^{\prime \prime}
\end{array}\right) \cdots .
$$

\section{Shift and variance of a transition array $n \ell^{N+1} \rightarrow$ $n \ell^{N} n^{\prime} \ell^{\prime}$ of E2 lines}

To our knowledge [47, Harrison and Johnson 48, are the first who introduced the term "transition array" for the entire bunch of lines resulting from transitions between two configurations. The first experimental spectrum showing transition arrays was published by Edlén in 1947 [49]: it concerned transition elements in the XUV range and the spectra were generated by low-inductance discharge lamps. Since then, such arrays have been observed in a very large variety of spectra (see for instance [50, 51, 52, 53, 54). Although, in tokamak applications, E2 lines appear usually as isolated (only a few distinct lines are visible), it is also possible to estimate the global properties (strength-weighted moments) of a transition array of E2 lines. In the present section, we consider the case of inter-configuration E2 lines for the specific transition array $n \ell^{N+1} \rightarrow n \ell^{N} n^{\prime} \ell^{\prime}$ with $\ell^{\prime}-\ell=0, \pm 2$ and $\ell+\ell^{\prime} \geq 2$. The moments of the distribution of the line energies can be written as:

$$
\mathcal{M}_{n}=\frac{\sum_{a, b}[\langle b|H| b\rangle-\langle a|H| a\rangle]^{n}|\langle a|Q| b\rangle|^{2}}{\sum_{a, b}|\langle a|Q| b\rangle|^{2}}
$$


where $a$ and $b$ run over all the exact eigenstates of the Hamiltonian in configurations $C$ and $C^{\prime}$, respectively, and $Q$ is the $z$ component of the quadrupole transition operator. The first two moments were calculated by Bauche-Arnoult et al. 24 using the second-quantization techniques of Judd 46. The most useful quantities are the mean energy $\mathcal{M}_{1}$ and the variance $\sigma^{2}=\mathcal{M}_{2}-\mathcal{M}_{1}^{2}$ related to the spectral width of the UTA. For the mean energy of the array, it was found in Ref. 24] that

$$
\mathcal{M}_{1}=E_{C^{\prime}}-E_{C}+\delta E,
$$

where $E_{C}$ and $E_{C^{\prime}}$ are the average energies of the initial and final configurations respectively. There exists a shift between the weighted average energy of a transition array and the difference of average energies of the initial and final configurations. The shift $\delta E$ stems from the weighting factor $\left.\langle a|Q| b\rangle\right|^{2}$ and is non zero for transition arrays $n \ell^{N+1} \rightarrow n \ell^{N} n^{\prime} \ell^{\prime}$. It was shown in Ref. [24] that $\sigma^{2}$ can be written as

$$
\sigma^{2}=\sum_{i} c_{i}\left[\sum_{k, k^{\prime}} d_{i}\left(k, k^{\prime}, \ell, \ell^{\prime}, \cdots\right) \times e_{i}\left(n \ell, n^{\prime} \ell^{\prime}, \cdots\right)\right],
$$

where $c_{i}$ are numerical coefficients depending on the number of equivalent electrons $N, d_{i}\left(k, k^{\prime}, \ell, \ell^{\prime}, \cdots\right)$ are combinations of $3 n j(n=1,2$ and 3$)$ symbols independent of $N$ and $e_{i}\left(n \ell, n^{\prime} \ell^{\prime}, \cdots\right)$ are products of Slater integrals of ranks $k$ and $k^{\prime}$. In the following, $F_{C}^{k}$ and $F_{C^{\prime}}^{k}$ represent the direct Slater integrals in configurations $C$ and $C^{\prime}$, respectively, and $G_{C^{\prime}}^{k}$ the exchange Slater integral of $C^{\prime}$. As in Ref. 24, we define the quantities:

$$
\begin{aligned}
x & =N(N+1)(4 \ell-N)(4 \ell-N+1)=(N+1)(4 \ell-N) w, \\
y & =N(N-1)(4 \ell-N+1)(4 \ell-N+2)=(N-1)(4 \ell-N+2) w, \\
z & =N(N-1)(4 \ell-N)(4 \ell-N+1)=(N-1)(4 \ell-N) w, \\
u & =N(4 \ell-N)(4 \ell-N+1)=(4 \ell-N) w, \\
v & =N(N-1)(4 \ell-N+1)=(N-1) w, \\
w & =N(4 \ell-N+1) .
\end{aligned}
$$

In the following, in agreement with the convention of Ref. 24], indices $n$ and $n^{\prime}$ are omitted in the Slater integrals. The shift is given by:

$$
\delta E=N \frac{(2 \ell+1)\left(2 \ell^{\prime}+1\right)}{(4 \ell+1)}\left(\sum_{k \neq 0} f_{k} F_{C^{\prime}}^{k}\left(\ell \ell^{\prime}\right)+\sum_{k} g_{k} G_{C^{\prime}}^{k}\left(\ell \ell^{\prime}\right)\right)
$$

with

$$
f_{k}=\left(\begin{array}{ccc}
\ell & k & \ell \\
0 & 0 & 0
\end{array}\right)\left(\begin{array}{ccc}
\ell^{\prime} & k & \ell^{\prime} \\
0 & 0 & 0
\end{array}\right)\left\{\begin{array}{ccc}
\ell & k & \ell \\
\ell^{\prime} & 2 & \ell^{\prime}
\end{array}\right\},
$$

and

$$
g_{k}=\left(\begin{array}{ccc}
\ell & k & \ell^{\prime} \\
0 & 0 & 0
\end{array}\right)^{2}\left(\frac{2}{5} \delta_{k, 2}-\frac{1}{2(2 \ell+1)\left(2 \ell^{\prime}+1\right)}\right),
$$


where $\delta_{i, j}$ represents a Kronecker's symbol. The variance can be written as

$$
\sigma^{2}=\sum_{i=1}^{7} H_{i} .
$$

As compared to Ref. [24, the term $H_{1}$ remains unchanged:

$$
\begin{aligned}
H_{1}= & \sum_{k \neq 0} \sum_{k^{\prime} \neq 0}\left(\frac{2 \delta_{k, k^{\prime}}}{(2 k+1)}-\frac{1}{(2 \ell+1)(4 \ell+1)}\right. \\
& \left.-(-1)^{k+k^{\prime}}\left\{\begin{array}{ccc}
\ell & \ell & k \\
\ell & \ell & k^{\prime}
\end{array}\right\}\right) \frac{(2 \ell+1)^{3}}{(4 \ell-1) 8 \ell(4 \ell+1)} \\
& \times\left(\begin{array}{ccc}
\ell & k & \ell \\
0 & 0 & 0
\end{array}\right)^{2}\left(\begin{array}{ccc}
\ell & k^{\prime} & \ell \\
0 & 0 & 0
\end{array}\right)^{2} \\
& \times\left[x F_{C}^{k}(\ell \ell) F_{C}^{k^{\prime}}(\ell \ell)+y F_{C^{\prime}}^{k}(\ell \ell) F_{C^{\prime}}^{k^{\prime}}(\ell \ell)-2 z F_{C}^{k}(\ell \ell) F_{C^{\prime}}^{k^{\prime}}(\ell \ell)\right]
\end{aligned}
$$

and one has

$$
\begin{aligned}
& H_{2}=\sum_{k \neq 0} \sum_{k^{\prime} \neq 0}\left(\frac{2(-1)^{k}}{(2 k+1)} \delta_{k, k^{\prime}}\left\{\begin{array}{rrr}
\ell^{\prime} & \ell^{\prime} & k \\
\ell & \ell & 2
\end{array}\right\}\right. \\
& -(-1)^{k}\left\{\begin{array}{ccc}
\ell & \ell & k^{\prime} \\
\ell & \ell & k
\end{array}\right\}\left\{\begin{array}{ccc}
\ell & \ell & k^{\prime} \\
\ell^{\prime} & \ell^{\prime} & 2
\end{array}\right\} \\
& \left.-\frac{1}{(2 \ell+1)(4 \ell+1)}\left\{\begin{array}{ccc}
\ell & \ell & k^{\prime} \\
\ell^{\prime} & \ell^{\prime} & 2
\end{array}\right\}\right) \frac{(2 \ell+1)^{3}\left(2 \ell^{\prime}+1\right)}{(4 \ell-1) 2 \ell(4 \ell+1)} \\
& \times\left(\begin{array}{ccc}
\ell & k & \ell \\
0 & 0 & 0
\end{array}\right)^{2}\left(\begin{array}{ccc}
\ell^{\prime} & k^{\prime} & \ell^{\prime} \\
0 & 0 & 0
\end{array}\right)\left(\begin{array}{ccc}
\ell & k^{\prime} & \ell \\
0 & 0 & 0
\end{array}\right) \\
& \times\left[u F_{C}^{k}(\ell \ell) F_{C^{\prime}}^{k^{\prime}}\left(\ell \ell^{\prime}\right)+v F_{C^{\prime}}^{k}(\ell \ell) F_{C^{\prime}}^{k^{\prime}}\left(\ell \ell^{\prime}\right)\right] \text {, } \\
& H_{3}=\sum_{k \neq 0} \sum_{k^{\prime}}\left[-2\left\{\begin{array}{ccc}
k & k^{\prime} & 2 \\
\ell^{\prime} & \ell & \ell
\end{array}\right\}+\left\{\begin{array}{ccc}
\ell^{\prime} & \ell^{\prime} & k \\
\ell & \ell & 2
\end{array}\right\}\left\{\begin{array}{ccc}
\ell^{\prime} & \ell^{\prime} & k \\
\ell & \ell & k^{\prime}
\end{array}\right\}\right. \\
& \left.-\frac{1}{(2 \ell+1)(4 \ell+1)}\left(\frac{2}{5} \delta_{k^{\prime}, 2}-\frac{1}{\left(2 \ell^{\prime}+1\right)}\right)\right] \frac{(2 \ell+1)^{3}\left(2 \ell^{\prime}+1\right)}{(4 \ell-1) 2 \ell(4 \ell+1)} \\
& \times\left(\begin{array}{ccc}
\ell & k^{\prime} & \ell^{\prime} \\
0 & 0 & 0
\end{array}\right)^{2}\left(\begin{array}{ccc}
\ell & k & \ell \\
0 & 0 & 0
\end{array}\right)^{2} \\
& \times\left[u F_{C}^{k}(\ell \ell) G_{C^{\prime}}^{k^{\prime}}\left(\ell \ell^{\prime}\right)+v F_{C^{\prime}}^{k}(\ell \ell) G_{C^{\prime}}^{k^{\prime}}\left(\ell \ell^{\prime}\right)\right] \text {, }
\end{aligned}
$$




$$
\begin{aligned}
H_{4}= & \sum_{k \neq 0} \sum_{k^{\prime} \neq 0}\left(\frac{2 \delta_{k, k^{\prime}}}{\left(2 \ell^{\prime}+1\right)(2 k+1)}-\left\{\begin{array}{ccc}
\ell & \ell & k \\
\ell & 2 & \ell^{\prime} \\
k^{\prime} & \ell^{\prime} & \ell^{\prime}
\end{array}\right\}\right. \\
& \left.-\frac{1}{(4 \ell+1)}\left\{\begin{array}{ccc}
\ell & \ell^{\prime} & 2 \\
\ell^{\prime} & \ell & k
\end{array}\right\}\left\{\begin{array}{ccc}
\ell & \ell^{\prime} & 2 \\
\ell^{\prime} & \ell & k^{\prime}
\end{array}\right\}\right) \frac{(2 \ell+1)^{2}\left(2 \ell^{\prime}+1\right)^{2}}{4 \ell(4 \ell+1)} \\
& \times\left(\begin{array}{ccc}
\ell & k & \ell \\
0 & 0 & 0
\end{array}\right)\left(\begin{array}{ccc}
\ell & k^{\prime} & \ell \\
0 & 0 & 0
\end{array}\right)\left(\begin{array}{ccc}
\ell^{\prime} & k & \ell^{\prime} \\
0 & 0 & 0
\end{array}\right)\left(\begin{array}{ccc}
\ell^{\prime} & k^{\prime} & \ell^{\prime} \\
0 & 0 & 0
\end{array}\right) \\
& \times w F_{C^{\prime}}^{k}\left(\ell \ell^{\prime}\right) F_{C^{\prime}}^{k^{\prime}}\left(\ell \ell^{\prime}\right),
\end{aligned}
$$

$$
\begin{aligned}
H_{5}= & \sum_{k} \sum_{k^{\prime}}\left[\frac{2 \delta_{k, k^{\prime}}}{\left(2 \ell^{\prime}+1\right)(2 k+1)}-\left\{\begin{array}{ccc}
\ell & \ell^{\prime} & k \\
\ell^{\prime} & 2 & \ell \\
k^{\prime} & \ell & \ell^{\prime}
\end{array}\right\}\right. \\
& \left.-\frac{1}{(4 \ell+1)}\left(\frac{2}{5} \delta_{k, 2}-\frac{1}{\left(2 \ell^{\prime}+1\right)}\right)\left(\frac{2}{5} \delta_{k^{\prime}, 2}-\frac{1}{2 \ell^{\prime}+1}\right)\right] \\
& \times \frac{(2 \ell+1)^{2}\left(2 \ell^{\prime}+1\right)^{2}}{4 \ell(4 \ell+1)}\left(\begin{array}{ccc}
\ell & k & \ell^{\prime} \\
0 & 0 & 0
\end{array}\right)^{2}\left(\begin{array}{ccc}
\ell & k^{\prime} & \ell^{\prime} \\
0 & 0 & 0
\end{array}\right)^{2} \\
& \times w G_{C^{\prime}}^{k}\left(\ell \ell^{\prime}\right) G_{C^{\prime}}^{k^{\prime}}\left(\ell \ell^{\prime}\right),
\end{aligned}
$$

and the last term (for the electrostatic part):

$$
\begin{aligned}
H_{6}= & \sum_{k \neq 0} \sum_{k^{\prime}}\left[\frac{(-1)^{k}}{\left(2 \ell^{\prime}+1\right)}\left\{\begin{array}{ccc}
\ell^{\prime} & \ell^{\prime} & k \\
\ell & \ell & k^{\prime}
\end{array}\right\}\right. \\
& -2(-1)^{k}\left\{\begin{array}{ccc}
k & k^{\prime} & 2 \\
\ell & \ell^{\prime} & \ell^{\prime}
\end{array}\right\}\left\{\begin{array}{ccc}
k & k^{\prime} & 2 \\
\ell^{\prime} & \ell & \ell
\end{array}\right\}-\frac{1}{(4 \ell+1)} \\
& \left.\times\left\{\begin{array}{ccc}
\ell & \ell^{\prime} & 2 \\
\ell^{\prime} & \ell & k
\end{array}\right\}\left(\frac{2}{5} \delta_{k^{\prime}, 2}-\frac{1}{\left(2 \ell^{\prime}+1\right)}\right)\right] \frac{(2 \ell+1)^{2}\left(2 \ell^{\prime}+1\right)^{2}}{2 \ell(4 \ell+1)} \\
& \times\left(\begin{array}{ccc}
\ell & k & \ell \\
0 & 0 & 0
\end{array}\right)\left(\begin{array}{ccc}
\ell^{\prime} & k & \ell^{\prime} \\
0 & 0 & 0
\end{array}\right)\left(\begin{array}{ccc}
\ell & k^{\prime} & \ell^{\prime} \\
0 & 0 & 0
\end{array}\right)^{2} \\
& \times w F_{C^{\prime}}^{k}\left(\ell \ell^{\prime}\right) G_{C^{\prime}}^{k^{\prime}}\left(\ell \ell^{\prime}\right) .
\end{aligned}
$$

The spin-orbit contribution is the same as for the E1 case [24]: 


$$
\begin{aligned}
H_{7}= & (N+1)(4 \ell-N+1) \frac{\ell(\ell+1)}{4(4 \ell+1)} \zeta_{C}^{2}(n \ell)+ \\
& +N(4 \ell-N+2) \frac{\ell(\ell+1)}{4(4 \ell+1)} \zeta_{C^{\prime}}^{2}(n \ell)+\frac{\ell^{\prime}\left(\ell^{\prime}+1\right)}{4} \zeta_{C^{\prime}}^{2}\left(n^{\prime} \ell^{\prime}\right) \\
& -N(4 \ell-N+1) \frac{\ell(\ell+1)}{2(4 \ell+1)} \zeta_{C}(n \ell) \zeta_{C^{\prime}}(n \ell) \\
& -(4 \ell-N+1) \frac{\ell(\ell+1)+\ell^{\prime}\left(\ell^{\prime}+1\right)-2}{4(4 \ell+1)} \zeta_{C}(n \ell) \zeta_{C^{\prime}}\left(n^{\prime} \ell^{\prime}\right) \\
& -N \frac{\ell(\ell+1)+\ell^{\prime}\left(\ell^{\prime}+1\right)-2}{4(4 \ell+1)} \zeta_{C^{\prime}}(n \ell) \zeta_{C^{\prime}}\left(n^{\prime} \ell^{\prime}\right),
\end{aligned}
$$

where $\zeta_{C}(n \ell)$ and $\zeta_{C^{\prime}}(n \ell)$ are the spin-orbit integrals in the respective configurations $C$ and $C^{\prime}$. Higher moments can also be calculated [26, 29, 30, 31, 55, which requires to use another modeling function than the Gaussian. The Generalized Gaussian method [56] can be applied, but the Normal Inverse Gaussian was shown to provide a better depiction of the array when the first four moments are known [57, 58].

The range of energy of the levels of the upper configuration $C^{\prime}$ responsible for the preferential emission constitutes an "emissive zone" 34. In the same way as for an E1 UTA, the shift and width of the emissive zone of an E2 UTA are deduced from those of the complete UTA by restricting them to the radial parameters related to the upper configuration of the array.

\section{Conclusion}

We propose analytical expressions for the number of electric-quadrupole (E2) lines both in the inter- and intra-configuration cases. The resulting formulas are based on three different modelings of the distribution of the angularmomentum projection $M$ (Gaussian, fourth-order Gram-Charlier and Generalized Gaussian). The Gram-Charlier modeling gives satisfactory results but, for high- $\ell$ electrons, the Generalized-Gaussian distribution is more accurate (as for E1 lines, see Ref. [42]). The results are better in the intra- than in the inter-configuration case, which can be explained by the fact that the number of lines between two different configurations is evaluated with averaged parameters (variance and kurtosis of the distribution of the angular-momentum projection $M)$. We also provide the expression of the generalized $J$-file sum rule giving the total strength of the lines arising from a given level of the upper configuration, together with the formula for the average strength-weighted position and variance of E2 lines. The next step will consist in investigating the statistics of the amplitudes and strengths of E2 lines.

\section{Appendix A: Boundary effect on the number of lines of an array}

At very small values of $J$, we can linearize the exponential in Eq. (27), i.e. 


$$
Q_{C}(J)=K_{C} \times(2 J+1)
$$

and

$$
Q_{C^{\prime}}(J)=K_{C^{\prime}} \times(2 J+1),
$$

where $K_{C}$ and $K_{C^{\prime}}$ do not depend on $J$.

(i) Inter-configuration case:

In the inter-configuration case, the error committed in considering that $\epsilon^{\prime}\left(\frac{1}{2}\right)=0$ in Eq. (15) is equal to

$$
\Delta_{\epsilon, \text { inter }}=5 \int_{-1 / 2}^{0} Q_{C}(J) Q_{C^{\prime}}(J) d J
$$

Using the linearized expressions (63) and (64), one obtains

$$
\Delta_{\epsilon, \text { inter }} \approx \frac{5}{6}\left(K_{C}+K_{C^{\prime}}\right)
$$

(ii) Intra-configuration case:

In the intra-configuration case, the error committed in considering that $\epsilon^{\prime}\left(\frac{1}{2}\right)=0$ in Eq. (15) is equal to

$$
\Delta_{\epsilon, \text { intra }}=3 \int_{-1 / 2}^{0} Q_{C}(J) Q_{C^{\prime}}(J) d J
$$

Using the linearized expressions (63) and (64), one obtains

$$
\Delta_{\epsilon, \text { intra }} \approx \frac{K_{C}+K_{C^{\prime}}}{2} .
$$

\section{Appendix B: Estimation of $J_{\min }$}

Usually, $J_{\min }$ is equal to 0 (for integer values) or to $1 / 2$ (for half-integer values). However, in some circumstances [42] (for instance in the case of configurations containing an electron in an orbital with a high angular momentum $\ell$ ), the distribution $P(M)$ exhibits a plateau, for which we showed that the Generalized Gaussian in a good aproximation [42. In that case, $J_{\text {min }}$ differs from 0 and $1 / 2$. An estimation of $J_{\min }$ can be obtained through the relation:

$$
Q(J) \geq \frac{1}{2}
$$

Using the expression of $Q(J)$ given in Eq. (34), we find

$$
J_{\min } \approx \lambda \sqrt{v}\left(\frac{1-\nu}{\nu}\right) W\left[\frac{\nu}{1-\nu} \Lambda^{\frac{\nu}{\nu-1}}\right]^{1 / \nu}-\frac{1}{2}
$$

with 


$$
\Lambda=\frac{\lambda^{2} v_{C}}{\nu^{2} g_{C}} \Gamma\left(\frac{1}{\nu}\right)
$$

and $x \mapsto W[x]$ represents Lambert's function, solution of $x e^{x}=y$. The function $W$ can be expanded as [59, 60, 61, 62]:

$$
W(x)=\ln x-\ln (\ln x)+\sum_{k=0}^{\infty} \sum_{m=1}^{\infty} c_{k m} \frac{[\ln (\ln x)]^{m}}{(\ln x)^{k+m}}
$$

where

$$
c_{k m}=\frac{(-1)^{k}}{m !} S[k+m, k+1]
$$

$S[p, q]$ being Stirling number of the first kind [63, 64, also denoted $S_{p}^{(q)}, s(p, q)$ or $\left[\begin{array}{l}p \\ q\end{array}\right]$. Stirling numbers can be obtained by recursion relations [60, and an explicit expression was provided by Karanicoloff 65. However, since we are only interested in an approximate formula, the first two terms $\ln x-\ln (\ln x)$ are sufficient.

\section{Appendix C: Number of Auger amplitudes}

Auto-ionization from a state that involves a hole in an inner subshell of the core is known as the Auger [66] effect, and the ejected electron is called an Auger electron. The term "auto-ionization" (applied to levels produced by excitation of loosely bound electrons) was coined by Shenstone 67. In the non-relativistic approximation the amplitude of Auger transitions is equal to the reduced matrix element of the Coulomb interaction operator $H_{c}$ :

$$
\left\langle C \gamma J\left\|H_{c}\right\| C^{\prime} \gamma^{\prime} J^{\prime} \epsilon \ell j J\right\rangle=\sqrt{2 J+1}\left\langle C \gamma J\left|H_{c}\right| C^{\prime} \gamma^{\prime} J^{\prime} \epsilon \ell j J\right\rangle,
$$

where $C$ is the configuration of an atom, $J$ is the quantum number of total angular momentum, $\gamma$ denotes all the additional quantum numbers and $\epsilon$ is the energy of the Auger electron. In intermediale coupling, the number of Auger amplitudes for a given channel $\epsilon_{\ell}$ or $\epsilon_{\ell j}$ can be obtained as the number of reduced matrix elements of the scalar operator acting between two configurations:

$$
N_{\text {Auger }}\left(C-C^{\prime}\right) \approx \int_{0}^{\infty} Q_{C}(J) Q_{C^{\prime \prime}}(J) d J
$$

where $C^{\prime \prime}$ denotes $C^{\prime} \epsilon \ell$ and $C^{\prime}$ is the final configuration of the ion. In the configuration $C^{\prime \prime}$, the contribution of the Auger electron to the variance and kurtosis of the distribution $P(M)$ is equal to the one of an electron of the discrete spectrum $v(\epsilon \ell)=v(\ell)$, where $v(\ell)$ is defined in Eq. (3) and

$$
\mu_{4}(\epsilon \ell)=\frac{1}{240}(2 \ell+1)\left(48 \ell^{4}+96 \ell^{3}+152 \ell^{2}+104 \ell+15\right) .
$$

It was shown by Kynienè et al. 44 that the Auger transition

$$
\ell_{1}^{4 \ell_{1}+1} \ell_{2}^{N_{2}} \ell_{3}^{N_{3}} \rightarrow \ell_{1}^{4 \ell_{1}+2} \ell_{2}^{N_{2}-1} \ell_{3}^{N_{3}-1} \epsilon \ell
$$


does not change under the replacements $N_{2} \rightarrow 4 \ell_{2}+4-N_{2}$ and $N_{3} \rightarrow 4 \ell_{3}+$ $4-N_{3}$. In the same way, the transition

$$
\ell_{1}^{4 \ell_{1}+1} \ell_{2}^{N_{2}} \rightarrow \ell_{1}^{4 \ell_{1}+2} \ell_{2}^{N_{2}-2} \epsilon \ell .
$$

does not change under the replacement $N_{2} \rightarrow 4 \ell_{2}+4-N_{2}$. This is a consequence of complementarity, and can be explained by the fact that the recoupling does not change the number of matrix elements [44]. Here also, since

$$
\begin{aligned}
& v\left(\ell_{1}^{4 \ell_{1}+1}\right)+v\left(\ell_{2}^{N_{2}}\right)+v\left(\ell_{1}^{4 \ell_{1}+2}\right)+v\left(\ell_{2}^{N_{2}-2}\right)+v(\epsilon \ell) \\
= & v\left(\ell_{1}^{4 \ell_{1}+1}\right)+v\left(\ell_{2}^{4 \ell_{2}+4-N_{2}}\right)+v\left(\ell_{1}^{4 \ell_{1}+2}\right)+v\left(\ell_{2}^{4 \ell_{2}+2-N_{2}}\right)+v(\epsilon \ell),
\end{aligned}
$$

we set $v=\left(v_{C}+v_{C^{\prime}}\right) / 2$ and, in order to obtain an expression symmetrical in $\mathrm{C}$ and $C^{\prime}, \alpha_{4}=\left(\alpha_{4, C}+\alpha_{4, C^{\prime}}\right) / 2$. Using the Gaussian expression of $P(M)$ (see Eq. (26), Kynienè et al. obtained [4]:

$$
N_{\text {Auger }}\left(C-C^{\prime}\right) \approx \frac{g_{C} g_{C^{\prime}}}{8 \sqrt{\pi} v^{3 / 2}} .
$$

The Gram-Charlier modeling of $P(M)$ (see Eq. (30) leads to

$$
N_{\text {Auger }}\left(C-C^{\prime}\right) \approx g_{C} g_{C^{\prime}} \frac{1009+5 \alpha_{4}\left(-62+21 \alpha_{4}\right)}{8192 \sqrt{\pi} v^{3 / 2}},
$$

and using a Generalized-Gaussian approximation of $P(M)$, we find

$$
N_{\text {Auger }}\left(C-C^{\prime}\right) \approx g_{C} g_{C^{\prime}} \frac{1}{2^{2+1 / \nu} \lambda \Gamma\left(1+\frac{1}{\nu}\right) \sqrt{v}} .
$$

\section{References}

\section{References}

[1] Sobelman I I, Introduction to the theory of atomic spectra (Pergamon, New York, 1972).

[2] Charro E and Martín I 2003 Ap. J. 5851191.

[3] Charro E, López-Ferrero S and Martín I 2003 A $\&$ \& 4406741.

[4] Klapisch M, Schwob J L, Finkenthal M, Fraenkel B S, Egert S, Bar-Shalom A, Breton C, DeMichelis C and Mattioli M 1978 Phys. Rev. Lett. 41403.

[5] Fournier K B, Goldstein W H, May M and Finkenthal M 1996 Phys. Rev. A 53709.

[6] Isler R C 1984 Nucl. Fusion 241599.

[7] Cummings J, Cohen S, Hulse R, Post D, Redi M and Perkins J $1990 \mathrm{~J}$. Nucl. Matter $\mathbf{1 7 6} \& \mathbf{1 7 7} 916$. 
[8] Fournier K B 1998 At. Data Nucl. Data Tables 681.

[9] Quinet P 2012 J. Phys. B: At. Mol. Opt. Phys. 45025003.

[10] Neu R, Dux R, Kallenbach A, Pütterich T, Balden M, Fuchs J C, Herrmann A, Maggi C F, O’Mullane M, Pugno R, Radivojevic I, Rohde V, Sips A C C, Suttrop W, Whiteford A and the ASDEX Upgrade team 2005 Nucl. Fusion $4 \mathbf{5} 209$.

[11] Clementson J, Beiersdorfer P and Gu M F 2010 Phys. Rev. A 81012505.

[12] Ralchenko Yu, Tan J N, Gillaspy J D, Pomeroy J M and Silver E 2006 Phys. Rev. A $\mathbf{7 4} 042514$.

[13] Ralchenko Yu, Reader J, Pomeroy J M, Tan J N and Gillaspy J D $2007 \mathrm{~J}$. Phys. B: At. Mol. Opt. Phys. 403861.

[14] Bar-Shalom A, Oreg J and Goldstein W H 1995 Phys. Rev. E 514882.

[15] Miao J and Yuan J 2004 Phys. Rev. E 69017401.

[16] Upcraft L M, Harris J W O and Crowley B J B 2006 J. Quant. Spectrosc. Radiat. Transfer 99627.

[17] Gauthier J C, Geindre J P, Monier P, Luc-Koenig E, Wyart J F 1986 J. Phys. B 19 L385.

[18] Wyart J F, Bauche-Arnoult C, Gauthier J C, Geindre J P, Monier P, Klapisch M, Bar-Shalom A and Cohn A 1986 Phys. Rev. A 34, 701.

[19] Cowan R D, The Theory of Atomic Structure and Spectra (University of California Press, Berkeley, 1981).

[20] Moore C E, Bibliography on the Analyses of Optical Atomic Spectra, U. S. Natl. Bur. Stand. Special Publ. 306 (U. S. Govt. Printing Off., Washington D. C., 1968-69).

[21] Hagan L and Martin W C, Bibliography on Atomic Energy Levels and Spectra, July 1968 through June 1971, U. S. Natl. Bur. Stand. Special Publ. 363 (U. S. Govt. Printing Off., Washington D. C., 1972).

[22] Charro E and Martin I, Int. J. Quant. Chem. 90, 403 (2002).

[23] Ginocchio J N 1973 Phys. Rev. C 8135.

[24] Bauche-Arnoult C, Bauche J and Klapisch M 1979 Phys. Rev. A 202424.

[25] Bauche-Arnoult C, Bauche J and Klapisch M 1982 Phys. Rev. A 252641.

[26] Bauche-Arnoult C, Bauche J and Klapisch M 1984 Phys. Rev. A 303026.

[27] Bauche-Arnoult C, Bauche J and Klapisch M 1985 Phys. Rev. A 312248.

[28] Bauche J and Bauche-Arnoult C 1990 Comput. Phys. Rep. 121.

[29] Karazija R, Sums of Atomic Quantities and Mean Characteristics of Spectra, (Mokslas, Vilnius, 1991), in russian. 
[30] Karazija R 1991 Acta Phys. Hungarica 70367.

[31] Karazija R 1992 Acta Phys. Hungarica 72305.

[32] Kučas S and Karazija R 1993 Phys. Scr. 47754.

[33] Karazija R and Kučas S 1995 Lith. J. Phys. 35155.

[34] Bauche J, Bauche-Arnoult C, Luc-Koenig E, Wyart J F and Klapisch M 1983 Phys. Rev. A 28829.

[35] Bauche J, Bauche-Arnoult C and Fournier K B 2004 Phys. Rev. E 69 026403.

[36] Krasnitz A, M. Sc. Thesis, Hebrew University of Jerusalem, 1984.

[37] Klapisch M, Krasnitz A, Mandelbaum P, Bauche-Arnoult C and Bauche $\mathrm{J}$, New results of the unresolved transition arrays method, Eight International Colloquium on Ultraviolet and X-ray Spectroscopy of Astrophysical and Laboratory Plasmas, Proceedings of IAU Colloq. 86, August $27^{t h}-29^{t h}$ 1984, Naval Research Laboratory, Washington D.C., p. 114.

[38] Bauche J and Bauche-Arnoult C 1987 J. Phys. B: At. Mol. Opt. Phys. 20 1659.

[39] Porcherot Q, Pain J C, Gilleron F and Blenski T 2011 High Energy Density Phys. 7234.

[40] Condon E U and Shortley G H, The theory of atomic spectra (Cambridge University Press, Cambridge, 1935).

[41] Kendall M G and Stuart A, Advanced Theory of Statistics (Hafner, New York, 1969), Vol. 1.

[42] Gilleron F and Pain J C 2009 High Energy Density Phys. 5320.

[43] Ezawa H and Leventhal M 1975 J. Phys. B 81824.

[44] Kynienė A, Karazija R and Jonauskas V 2002 J. Electron Spectrosc. Relat. Phenom. 122181.

[45] Rosenzweig N and Porter C E 1961 Phys. Rev. 123853.

[46] Judd B R, Second Quantization and Atomic Spectroscopy (Johns Hopkins University, Baltimore, 1967).

[47] Bauche J, Bauche-Arnoult C and Klapisch M 1988 Adv. At. Mol. Phys. 23 131.

[48] Harrison G R and Johnson M H 1931 Phys. Rev. 38757.

[49] Edlén B 1947 Physica (Utrecht) 13545.

[50] Klapisch M, Meroz E, Mandelbaum P, Zigler A, Bauche-Arnoult C and Bauche J 1982 Phys. Rev. A 252391.

[51] Svendsen W and O'Sullivan G 1994 Phys. Rev. A 503710. 
[52] Palmeri P and E. Biémont E 1996 Phys. Rev. A 541337.

[53] Kučas S, Jonauskas V and Karazija R 2005 Nucl. Instr. Meth. Phys. Res. B 235155.

[54] Loisel G, Arnault P, Bastiani-Ceccotti S, Blenski T, Caillaud T, Fariaut J, Fölsner W, Gilleron F, Pain J C, Poirier M, Reverdin C, Silvert V, Thais F, Turck-Chièze S and Villette B 2009 High Energy Density Phys. 5173.

[55] Jonauskas V, Kučas S, Karazija R and Norrington P H 2007 Phys. Scr. 75 237.

[56] Gilleron F, Pain J C, Bauche J and Bauche-Arnoult C 2008 Phys. Rev. E 77026708 .

[57] Pain J C, Gilleron F, Bauche J and Bauche-Arnoult C 2009 High Energy Density Phys. 5294.

[58] Pain J C, Gilleron F, Bauche J and Bauche-Arnoult C 2010 High Energy Density Phys. 6356.

[59] Lambert J H 1758 Acta Helvetica 3128.

[60] Comtet L 1970 C. R. Acad. Sci. Paris 2701085.

[61] Jeffrey D J, Corless R M, Hare D E G and Knuth D E 1995 C. R. Acad. Sci. Paris 3201449.

[62] Hassani M 2005 Approximation of the Lambert W Function, RGMIA Research Report Collection, 8 Article 12.

[63] Comtet L, Advanced combinatorics, (D. Reidel Publishing Company, 1974).

[64] Mitrinović D S and Doković D 1960 C. R. Acad. Sci. 2502110.

[65] Karanicoloff Chr 1961 Publications de la faculté d'électrotechnique de l'université à Belgrade $\mathbf{6 7}$ 9, in French.

[66] Auger P 1925 J. Phys. radium 6205.

[67] Shenstone A G 1931 Phys. Rev. 38873. 